\title{
Treinamento
}

\section{Diferentes intervalos entre séries e sua influência no volume total dos exercícios resistidos}

\author{
Roberto Simão - CREF 2486 G/RJ \\ Escola de Educação Física e Desporto/UFRJ \\ robertosimao@ig.com.br

\section{João Antônio Almeida Alves de Souza \\ Universidade Católica de Petrópolis \\ joao@click21.com.br}

\section{Sidney Cavalcante - CREF 2042 G/RJ} \\ Universidade Católica de Petrópolis \\ Universidade Gama Filho - CEPAC \\ sidneycavalcante@compuland.com.br
}

\author{
Humberto Miranda - CREF 4313 G/RJ \\ Universidade Gama Filho - CEPAC \\ humbertomiranda01@pop.com.br \\ Luis Viveiros - CREF 012371 G/RJ \\ Universidade Gama Filho - CEPAC \\ luisviveiros@ideativa.org.br
Alex Souto Maior - CREF 12371 G/RJ
Universidade Gama Filho - CEPAC
Programa de Bioengenharia/UNIVAP
alex.souto@ig.com.br

SIMÃO, R.; SOUZA, J.A.A.A.; CAVALCANTE, S.; MIRANDA, H.; VIVEIROS, L.; MAIOR, A.S. Diferentes intervalos entre séries e sua influência no volume total dos exercícios resistidos. Fitness \& Performance Journal, v. 5, n² 2, p. 76-80, 2006.

\begin{abstract}
Resumo - Este estudo teve como objetivo comparar os efeitos de três diferentes tempos de intervalos de descanso entre séries com o volume total completado, em três séries com cargas para 10RM nos exercícios Leg Press $45^{\circ}$ e Supino Horizontal. Foram avaliados 11 sujeitos do sexo masculino, com idade de $21 \pm 3$ anos; massa corporal 72,6 $6 \pm 12,2 \mathrm{~kg}$; e altura $174 \pm 5,8 \mathrm{~cm}$. Todos os sujeitos realizaram duas sessões de testes de carga máxima para 10RM e três sessões de treino, durante as quais três séries de Leg Press $45^{\circ}$ e Supino Horizontal foram realizadas com cargas para 10RM. Durante cada sessão de treino foram feitos intervalos de descanso de 1, 3 e 5 minutos entre as séries. O volume total de exercício foi definido como sendo o total de repetições completadas somando-se as três séries, em cada exercício e em cada tempo de descanso. A análise estatística foi conduzida separadamente por uma ANOVA para medidas repetidas em cada exercício. Os intervalos de descanso de 3 e 5 minutos resultaram em um maior volume completado sem diferença significativa entre si, mas com diferenças em relação a 1 minuto. O volume total de repetições máximas por exercício, em todas as séries executadas, é afetado pelo tempo de recuperação, principalmente em 1 minuto de intervalo.
\end{abstract}

Palavras-chave: força, número de séries, intervalo, descanso, volume

\section{Endereço para correspondência:}

Rua Desenhista Luís Guimarães, 260 - apt 601 . Barra da Tijuca - Rio de Janeiro/RJ

Data de Recebimento: Novembro/2005

Data de Aprovação: Janeiro/2006

Copyright@ 2006 por Colégio Brasileiro de Atividade Física Saúde e Esporte. 
Different rest intervals between series and their influence in the total volume of resisted exercices

The objective of this study was to compare the effects of three different times of rest intervals between series with the completed total volume, in three series with loads for 10RM in the exercises Leg Press $45^{\circ}$ and Chest Press. It was appraised 11 male subjects (age $21 \pm 3$ years; mass 72,6 \pm 12,2 kg; height $174 \pm 5,8$ $\mathrm{cm})$. All the subjects accomplished two sessions of tests using maximum load for $10 R M$ and three training sessions, during which there were three series of Leg Press $45^{\circ}$ and Chest Press with loads for 10RM. During each training session there were rest intervals of 1, 3 and 5 minutes between the series. The total volume of exercise was defined as being the total of completed repetitions being added the three series, in each exercise and in every time of rest. The statistical analysis was driven separately by an ANOVA for repeated measures in each exercise. The rest intervals of 3 and 5 minutes resulted in a larger volume completed without significant difference amongst themselves, but with differences in relation to 1 minute. The total volume of maximum repetitions for exercise, in all of the executed series, is affected by the time of recovery, mainly in 1 minute of interval.

Keywords: strength, number of series, interval, rest, volume
Los intervalos diferentes descansan entre la serie e influencian en el volumen total de los ejercicios resistidos

El objetivo del estudio era comparar los efectos de tres tiempos diferentes de intervalos del resto entre la serie con el volumen total completado, en tres serie con las cargas para 10RM de los ejercicios Prensa en la Pierna $45^{\circ}$ y Supino Horizontal. Ellos se estimaron 11 varón de los asuntos (la edad $21 \pm 3$ años; la masa corpórea $72,6 \pm 12,2 \mathrm{~kg}$; la altura $174 \pm 5,8$ centímetro). Todos los asuntos lograron dos sesiones de pruebas de carga máxima para 10RM tres sesiones de entrenamiento. Durante cada sesión de entrenamiento ellos se hicieron los intervalos de resto de 1, 3 y 5 minutos de resto entre la serie. El volumen total de ejercicio se definió como ser el total de repeticiones completadas que se agregan la tres serie, en cada ejercicio y en cada vez de resto. El análisis estadístico se manejó separadamente por un ANOVA para las medidas repetidas en cada ejercicio. Los intervalos de resto de 3 y 5 minutos producían un volumen más grande completado sin la diferencia significante entre ellos, pero con las diferencias respecto a 1 minuto. El volumen total de repeticiones máximas para el ejercicio, en toda la serie ejecutada, es afectado cuando de recuperación principalmente en 1 minuto de intervalo.

Palabras-Clave: fuerza, número de la serie, intervalo, resto, volumen

\section{INTRODUÇÃO}

Diversas são as variáveis que podem influenciar na elaboração do treinamento de força. Entre as variáveis metodológicas de prescrição podemos citar a intensidade da carga, o número de repetições, a ordem dos exercícios, a freqüência semanal, o intervalo entre as séries e sessões, e o número de séries (ACSM, 2002). Tais variáveis, quando manipuladas adequadamente, permitem atingir os objetivos almejados em um programa de exercícios resistidos (ER), podendo ser estruturados com ênfase na resistência muscular, hipertrofia, força, ou potência muscular (ACSM, 2002; SIMÃO et al., 2005).

Uma das variáveis de grande importância na elaboração dos ER são os intervalos de recuperação entre as séries e exercícios. Os períodos de descanso têm influência na determinação do estresse do treino e no total da carga que pode ser manipulada. Alguns estudos (AHTIAINEN et al., 2005; MATUSZAK et al., 2003; PINCIVERO et al., 1997; WILLARDSON, BURKETT, 2004; WOODS et al., 2004) têm demonstrado que o tempo de intervalo entre as séries tem um efeito significativo sobre o volume total completado durante uma seqüência de um determinado exercício, o que conseqüentemente pode afetar o desenvolvimento da força e hipertrofia (AHTIAINEN et al., 2005; WEIR et al., 1994).

No entanto, os resultados ainda são escassos, não permitindo inferências consistentes sobre o tempo adequado de recuperação entre as séries e exercícios, para otimizarem objetivos diversificados na prescrição. Segundo FLECK \& KRAEMER (2004), a manipulação cuidadosa dos períodos de descanso é essencial para evitar que se coloque uma tensão inadequada e desnecessária no indivíduo durante o treinamento. Nesse contexto, além dos objetivos da prescrição, é provável que aspectos, como os distintos grupos musculares, a ordenação dos exercícios, os diferentes graus de aptidão física dos praticantes e os sistemas de treinamento adotados, possam influenciar na definição dos intervalos entre séries e exercícios.

Diferenças metodológicas, incluindo os métodos para detecção das cargas de treinamento, os percentuais de carga em que os indivíduos realizaram as repetições máximas, os exercícios selecionados e as populações estudadas, podem ser responsáveis pelas diferenças de resultados observadas nos distintos experimentos. Dessa forma, pode-se afirmar que as diferentes manipulações das variáveis de prescrição nos ER podem interferir na recuperação entre as séries. São poucos os estudos que investigaram a influência de diferentes intervalos de recuperação para grupos musculares distintos em uma mesma amostra.

Em adição, os resultados desses experimentos ainda parecem ser inconsistentes. Assim, o presente estudo tem como propósito verificar a influência de três diferentes intervalos de recuperação no volume total completado, aplicados em dois exercícios para grupos musculares distintos, realizados em 
três séries com cargas para 10 repetições máximas (10RM) em indivíduos treinados.

\section{MATERIAIS E MÉTODOS}

\section{Amestra}

Participaram deste estudo 11 homens (21 $\pm 3,4$ anos; 72 $\pm 12,3 \mathrm{~kg} ; 174 \pm 5,8 \mathrm{~cm})$, que não apresentavam contra indicações para aplicação do teste de 10RM. Todos os indivíduos eram fisicamente ativos, praticantes de ER há pelo menos dois anos, com freqüência mínima de três vezes semanais. Antes da coleta de dados, todos os indivíduos responderam negativamente ao questionário $P A R-Q$ e assinaram um termo de consentimento pós-informado, conforme a resolução do Conselho Nacional de Saúde (196/96).

\section{Teste de IORM}

Os dados foram coletados em dois dias distintos. Na primeira visita ao laboratório, depois de realizarem as medidas antropométricas, os indivíduos executaram o teste de 10RM (BACHLE, EARLE, 2000). Após a obtenção das cargas máximas no teste de 10RM, os indivíduos descansaram por 48 horas e foram reavaliados para obtenção da reprodutibilidade das cargas no teste de 10RM (segundo dia de teste). Para tanto, considerou-se como 10RM a maior carga estabelecida em ambos os dias. Nos intervalos entre as sessões de testes não foi permitida a realização de exercícios, visando não interferir nos resultados obtidos.

O procedimento de aplicação do teste 10RM foi na forma de delineamento alternado para o Supino Horizontal ( $\mathrm{SH}$ ) e Leg Press 450 (LP). Os exercícios foram selecionados devido à sua disseminação em centros de treinamento e facilidade de execução. Além disso, optou-se por selecionar exercícios que envolviam diferentes grupamentos musculares, o que permitiu avaliar a influência dos distintos intervalos de recuperação nesses grupamentos.

Visando reduzir a margem de erro nos testes de 10RM, foram adotadas as seguintes estratégias:

a) instruções padronizadas foram fornecidas antes do teste, de modo que o avaliado estivesse ciente de toda a rotina que envolvia a coleta de dados;

b) o avaliado foi instruído sobre a técnica de execução do exercício;

c) o avaliador estava atento quanto à posição adotada pelo praticante no momento da medida, pois pequenas variações no posicionamento das articulações envolvidas no movimento poderiam acionar outros músculos, levando a interpretações errôneas dos escores obtidos;

d) estímulos verbais foram realizados a fim de manter alto o nível de estimulação;

e) os pesos adicionais utilizados no estudo foram previamente aferidos em balança de precisão.
Os intervalos entre as tentativas em cada exercício durante o teste de 10RM foram fixados entre dois a cinco minutos (BACHLE, EARLE, 2000). Após obtenção da carga em um determinado exercício, intervalos não inferiores há 10 minutos foram dados, antes de passar-se ao teste no exercício seguinte.

\section{Aplicação dos diferentes intervalos entre séries}

Uma vez determinada à carga de treinamento em 10RM, nas três sessões restantes, uma a cada semana, foram executadas três séries de SH e LP com cargas para 10RM em intervalos de descanso de 1, 3 e 5 minutos entre as séries. Foram dados 10 minutos de descanso passivo antes de começar a série de aquecimento para o próximo exercício. O procedimento de entrada alternada foi utilizado para determinar a ordem de execução dos exercícios e o tempo de intervalo de descanso utilizado em cada sessão de testes.

Antes das sessões de treinamento, os sujeitos realizaram o aquecimento em cada exercício, sendo este de 15 repetições com 50\% da carga para 10RM. Os exercícios em todas as sessões de treinamento foram realizados com três séries até a fadiga com cargas para 10RM. Foi permitido aos sujeitos que continuassem com seus treinos durante a realização da pesquisa, com as seguintes exceções: (a) eles não deveriam realizar os exercícios de LP e SH em seus treinos pessoais e (b) eles não deveriam fazer exercícios 48 horas antes dos dias marcados para a realização do teste.

\section{Tratamento Estatístico}

Aplicou-se uma ANOVA de uma entrada para medidas repetidas, com intuito de verificar o volume total de repetições por exercício obtidos nas três séries nos três diferentes tempos de intervalo. A determinação da reprodutibilidade nas cargas para 10RM foi realizada através do coeficiente de correlação intra-classe, adicionado ao teste t de student pareado. O nível de significância adotado foi de $5 \%$.

\section{RESULTADOS}

Inicialmente são ilustrados os resultados referentes à reprodutibilidade das cargas obtidas nos testes de 10RM. Como pode ser constatado, foram verificados elevados valores coeficientes de correlação intraclasse nos exercícios selecionados (SH r=0,94 e LP r=0,92). Em adição, o teste-t student pareado não demonstrou diferença significativa entre as cargas obtidas para 10RM em cada exercício $(p<0,05)$.

A seguir são apresentados os resultados referentes a cada intervalo de tempo nas diferentes séries em cada exercício. $O$ volume total de trabalho completado no $\mathrm{SH}$ apresentou diferenças significativas $(p<0,05)$ entre os intervalos de descanso de 1 e 3 minutos, e entre 1 e 5 minutos, já entre os intervalos de 3 e 5 minutos não houve diferença significativa (Figura 1). Os resultados encontrados para 0 LP em relação ao volume total de trabalho foram similares 


\section{TMezLi 1}

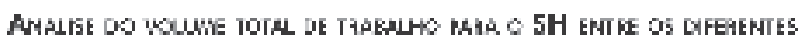

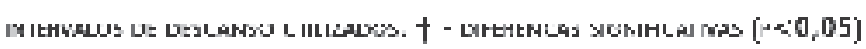

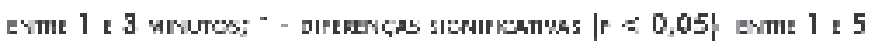
Nimuros

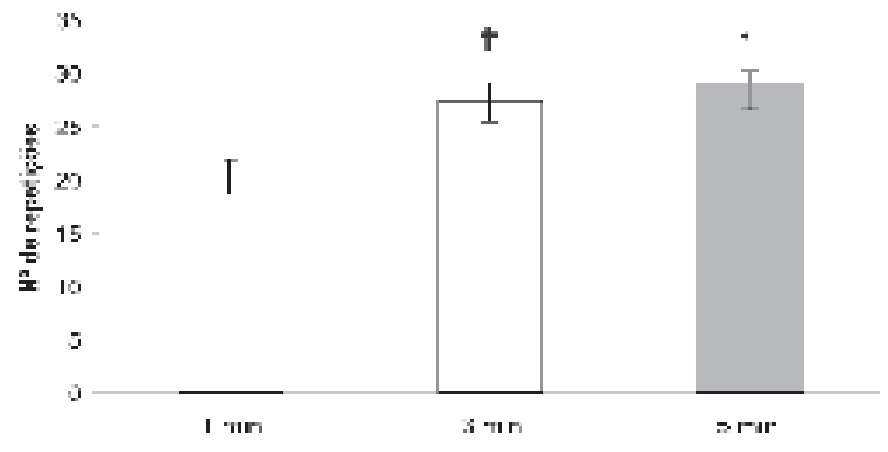

\section{TAreth 1}

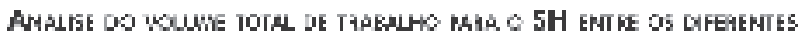

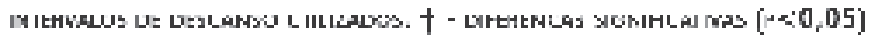

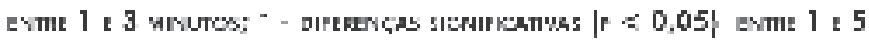
Ninuros

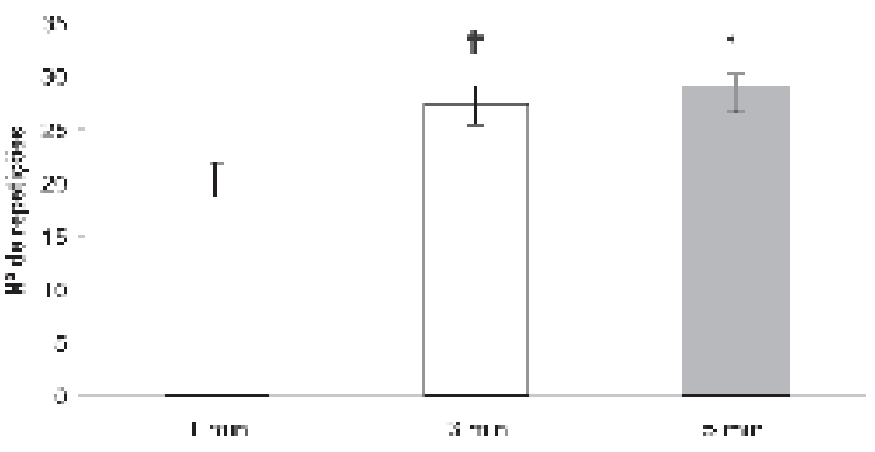

àqueles encontrados para o $\mathrm{SH}$, com significância $(\mathrm{p}<0,05)$ entre os intervalos de 1 e 3 minutos e entre 1 e 5 minutos (Figura 2).

\section{Discussão}

$\bigcirc$ objetivo do presente estudo foi investigar os efeitos de diferentes intervalos nos desempenhos da força em diferentes números de séries e exercícios. De acordo com o posicionamento do American College of Sports Medicine (ACSM, 2002) em relação ao treinamento de força para praticantes intermediários e avançados, devem ser utilizados intervalos entre 2 a 3 minutos para exercícios multi-articulares que envolvam massas musculares relativamente grandes.

posicionamento destaca que essa faixa de intervalo parece ser suficiente para provocar uma adequada recuperação entre as séries. Contudo, essa ainda é uma questão pouco explorada na literatura. No presente estudo, verificou-se que em todas as séries de repetições máximas, em todos os intervalos de recuperação investigados, houve redução no número total de repetições.
Normalmente, fisiculturistas utilizam intervalos curtos entre as séries, causando um elevado estresse muscular e promovendo talvez, uma hipertrofia acentuada (SIMÃO et al., 2005; TESH, LARSON, 1982) devido principalmente a uma maior liberação dos hormônios anabólicos. Períodos de intervalo curtos são acompanhados de considerável desconforto muscular, devido à oclusão do fluxo sangüíneo e produção de lactato (TESH, LARSON, 1982) e, conseqüentemente, diminuição da produção de força (WILLARDSON, BURKETT, 2004).

Entretanto, os levantadores de peso utilizam altas cargas de treinamento, mas com períodos de descanso elevados quando comparados aos fisiculturistas. Esse longo intervalo seria necessário para promover restabelecimento das funções orgânicas (KRAEMER et al., 1996), entre as quais podemos destacar a recuperação do sistema neural e energético. Normalmente, o período de intervalo compreende de 30-60 segundos para fisiculturistas e de 2-5 minutos para levantadores de peso (KRAEMER et al., 1987).

Os nossos resultados corroboram as evidências encontradas na literatura. $\bigcirc$ grupo aqui avaliado era composto de homens treinados e, mesmo assim, apresentou uma redução significativa no volume total de treinamento em ambos os exercícios de ER com 1 minuto de intervalo, em comparação aos com 3 e 5 minutos. Essa redução do volume total de treinamento foi decorrente de um alto grau de fadiga, que promoveu uma diminuição da produção de força e influenciou negativamente o número de repetições totais.

Entretanto, quando esses mesmos indivíduos realizaram os mesmos exercícios com 5 minutos de intervalo, uma maior produção de força foi obtida, resultando em um maior número de repetições em cada série, promovendo um maior volume total de treinamento. Vale ressaltar que, ao comparar 3 e 5 minutos em ambos os exercícios, não foram encontradas diferenças significativas; no entanto, observou-se um menor volume total em 3 minutos, quando comparado a 5 minutos.

WILLARDSON \& BURKETT (2004) avaliaram 15 homens experientes em treinamento de força, no supino e agachamento, em quatro séries de 8RM, com intervalos de 1, 2 e 5 minutos entre as séries. Os testes foram realizados em três dias diferentes, uma vez por semana. Foram encontradas diferenças significativas entre 1, 2 e 5 minutos de intervalo no supino: o intervalo de 5 minutos apresentou o maior volume completado, seguido do de 3 minutos e, por fim, do de 1 minuto.

Já no agachamento, os resultados se apresentaram similares, porém foram encontradas diferenças significativas nos volumes entre os intervalos de 1 e 5 minutos, e entre os de 2 e 5 minutos. Já entre os intervalos de 1 e 2 minutos não foram encontradas diferenças significativas. RICHMOND \& GODARD (2004)avaliaram 28 homens que executaram no 
supino duas séries a $75 \%$ de 1 RM até a exaustão voluntária, com intervalos de 1, 3 e 5 minutos entre as séries, em três dias diferentes. Foram encontradas diferenças significativas no volume completado no supino de 1 minuto de intervalo em relação ao de 3 e 5 minutos. Porém, não foram encontradas diferenças significativas entre os intervalos de 3 e 5 minutos. Assim, mesmo existindo diferenças metodológicas nos estudos supracitados, ambos demonstraram que um maior tempo de intervalo entre as séries permitiu um maior volume total de treinamento nos exercícios avaliados.

KRAEMER (1997), que avaliou os efeitos de intervalos de 1 -3 minutos entre as séries em $10 R M$ no supino e no leg press, em dias diferentes, em 20 jogadores de futebol americano da primeira divisão, verificou que o intervalo de 1 minuto entre as séries não foi suficiente para que se completassem as 10RM, com uma significativa redução no número total de repetições; porém, com intervalos de 3 minutos, se alcançou o número de repetições objetivado.

Em experimento realizado, ROBINSON et al. (1995), compararam o desempenho de 33 homens moderadamente treinados, em um programa de treinamento, durante cinco semanas com quatro sessões de treinamento semanais, em três diferentes tempos de intervalo (3 minutos, 90 segundos e 30 segundos). Verificou-se, através do teste de 1 RM no agachamento, que com um maior tempo de intervalo entre as séries, uma maior carga foi alcançada, o que gerou maiores ganhos de força no grupo de 3 minutos de intervalo. Em nosso experimento, na relação do somatório das repetições nas três séries em cada exercício, o intervalo de 1 minuto demonstrou resultados significativamente menores em todos os exercícios, quando comparado aos intervalos de 3 e 5 minutos (Tabela 1).

Esses nossos achados são muito similares aos encontrados nos estudos citados anteriormente (WILLARDSON, BURKETT, 2004; KRAEMER, 1997; ROBINSON et al., 1995). Ficou evidenciado que o volume por exercício foi significativamente diminuído ao se reduzir o tempo de intervalo, e que 1 minuto de intervalo não é suficiente para recompor os substratos energéticos ou sistema neural para se completar uma nova série, na mesma intensidade trabalhada.

Apesar de o intervalo de 3 minutos também não ter sido suficiente para mantermos as 10RM, o número de repetições alcançados foi significativamente superior ao intervalo de 1 minuto, mas sem significância em relação ao de 5 minutos. Podemos observar que um intervalo de 5 minutos em ambos os exercícios foi praticamente suficiente para a realização das $10 R M$ em ambos os exercícios.

\section{APLICAÇÕES PRÁtICAS}

$\bigcirc$ presente estudo verifica que o tempo de intervalo entre as séries é um fator de extrema importância ao se manipular uma sessão de treinamento, pois afeta diretamente o volu- me total desta, podendo influenciar assim nos ganhos de força. Ao compararmos os intervalos de 1, 3 e 5 minutos, percebe-se que o de 1 minuto não é suficiente para que os substratos energéticos e/ou o sistema neural sejam recuperados para completarmos a série seguinte, mantendo a mesma intensidade. Nos intervalos de 3 minutos obteve-se um maior volume na sessão de treinamento do que no de 1 minuto; no entanto, para uma restauração praticamente completa, um bom tempo de recuperação para os exercícios selecionados neste treinamento e nesta intensidade parece estar perto de 5 minutos.

Novos estudos envolvendo sessões de treinamento com diferentes volumes de treinamento, exercícios e com diferentes intervalos nos ER devem ser realizados, objetivando encontrar o melhor tempo para um maior volume de treino.

\section{BIBLIOGRAFIA}

AMERICAN COLLEGE OF SPORTS MEDICINE. Position stand: Progression models in resistance training for healthy adults. Medicine and Science in Sports Exercise, v.34, p.364-380, 2002.

AHTIAINEN, J.P.; PAKARINEN, A.; ALEN, M.; KRAEMER, W.J.; HÄKKINEN, $K$. Short vs. long rest period between the sets in hypertrophic resistance training: Influence on muscle strength, size, and hormonal adaptations in trained men. Journal of Strength and Conditioning Research, v.19, p.572$582,2005$.

BACHLE, T.R.; EARLE, R.W. Essentials of strength training and conditioning. Champaign: Human Kinetics, 2000.

FLECK, S.J.; KRAEMER, W.J. Designing resistance training programs. Champaign: Human Kinetics, 2004.

KRAEMER, W.J. A series of studies: The physiological basis for strength training in American football: Fact over philosophy. Journal of Strength and Conditioning Research, v.11, p.131-142, 1997.

KRAEMER, W.J.; FLECK, S.J.; EVANS, W.J. Strength and power training: physiological mechanisms of adaptation. Exercise Sport Science Review, v.24, p.363-397, 1996.

KRAEMER, W.J.; NOBLE, B.J.; CLARK, M.J.; CULVER, B.W. Physiologic responses to heavy-resistance exercise with very short rest period. International Journal Sports Medicine, v.8, p.247-252, 1987.

MATUSZAK, M.E.; FRY, A.C.; WEISS, L.W.; IRELAND, T.R.; MCKNIGHT, M.M. Effect of rest interval length on repeated 1 repetition maximum back squats. Journal of Strength and Conditioning Research, v.17, p.634-637, 2003.

PINCIVERO, D.M.; LEPHART, S.M.; KARUNAKARA, R.G. Effects of rest interval on isokinetic strength and functional performance after short term high intensity training. British Journal Sports Medicine, v.31, p.229-234, 1997.

RICHMOND, S.R.; GODARD, P.M. The effects of varied rest periods between sets to failure using the bench press in recreationally trained men. Journal of Strength and Conditioning Research, v. 18, p.846-849, 2004.

ROBINSON, J.M.; STONE, M.H.; JOHNSON, R.L.; PENLAND, C.M.; WARREN, B.J.; LEWIS, R.D. Effects of different weight training exercise/rest intervals on strength, power, and high intensity exercise endurance. Journal of Strength and Conditioning Research, v.9, p.216-221, 1995.

SIMÃO, R.; FARINATTI, P.T.V.; POLITO, M.D.; MAIOR, A.S.; FLECK, S.J. Influence of exercise order on the number of repetitions performed and perceived exertion during resistive exercises. Journal of Strength and Conditioning Research, v.11, p.152-156, 2005.

TESH, P.; LARSON, L. Muscle hypertrophy in bodybuilders. European Journal Apply Physiology, v.49, P.301-306, 1982.

WEIR, J.P.; WAGNER, L.L.; HOUSH, T.J. The effect of rest interval length on repeated maximal bench presses. Journal of Strength and Conditioning Research, v.8, p.58-60, 1994.

WILLARDSON, J.M.; BURKETT, L.N. A comparison of 3 different rest intervals on the exercise volume completed during a workout. Journal of Strength and Conditioning Research, v.19, p.23-26, 2005.

WOODS, S.; BRIDGE, T.; NELSON, D.; RISSE, K.; PINCIVERO, D.M. The effects of rest interval length on ratings of perceived exertion during dynamic knee extension exercise. Journal of Strength and Conditioning Research, v.18, n.540-545, 2004. 